\title{
Ab initio calculations of dissociation energy of water molecule on near-term quantum devices using the Pennylane interface to quantum hardware.
}

Ben Geoffrey A S

https://orcid.org/0000-0001-6545-6314

Email for correspondence : bengeof@gmail.com

\begin{abstract}
Fault-tolerant quantum computers may be far off, however solving real world quantum chemistry problems on near-term quantum devices is possible through the Pennylane which provides interface to use any of the quantum hardware provided by any of the quantum hardware providers such as IBM, Google or Microsoft. A demonstrated use of Pennylane to solve real world quantum chemical problems on near-term quantum devices is shown. Ab initio quantum chemical calculations of dissociation energy of water molecule on the near-term quantum hardware using the Pennylane interface yielded a dissociation energy comparable to experiment and therefore it is recommended that the quantum chemistry community use nearterm quantum devices to leverage quantum computing advantage to solve quantum chemical problems.
\end{abstract}




\section{Introduction}

The developers of quantum hardware such as IBM, Google, Microsoft provide programming interface to their hardware which go by names Qiskit, Cirq and Q\# respectively for the development of real-world applications that leverage the of quantum hardware using quantum algorithms [1-5]. Although fault-tolerant quantum computers may be far off, solving real world quantum chemistry problems using near-term quantum devices is possible through Pennylane which provides interface to use any of the quantum hardware providers mentioned above [6]. A demonstration of ab initio calculations of dissociation energy of water molecule on the nearterm quantum hardware using the Pennylane interface is presented in the work below.

\section{Methodology}

The strategy to solve the problem of dissociation energy of water molecule ab initio involves obtaining the ab initio energies of reactant and products and obtaining the difference between them. In this work we implement this strategy on the near-term quantum devices through the Pennylane interface. The strategy to solve this problem using Pennylane is to use the Variational Quantum Eigensolver(VQE) method [7-9]. A quantum circuit is setup which reflects the trial wave function of the ground state of the molecular Hamiltonian and a classical optimizer is used to optimize the circuit parameters to render the molecule's ground state energy. The input cartesian structure file for the molecules is downloaded from chemical structure libraries. The read_structure() method of the qchem module of Pennylane is used to read the input structure. The construction of the molecular Hamiltonian and mapping of the initial trial wave function which is to be optimized to the qubits is carried out by Pennylane using quantum chemistry drivers and the default driver option is PySCF [10]. It requires the charge, spin multiplicity, basis set, the valence electrons and orbitals to be specified. The mapping of the trial wave function to qubits of the quantum circuit is carried out using the 'jordan-wigner' mapping. The quantum circuit of entangled qubits required to optimize the ground state of water, oxygen and hydrogen involved 12, 8 and 4 qubits, respectively. The code and implementation are provided in the GitHub link shared below.

https://github.com/bengeof/PennylaneDissociationEnergyAb-Initio 


\section{Results and discussion}

The ground state energy of water obtained from 100 iterations of optimization is -74.59262921 Ha. and the final circuit parameters and other optimization details are given below.

Iteration $=0$, Energy $=-73.63914635 \mathrm{Ha}$

Iteration $=20$, Energy $=-74.20924302 \mathrm{Ha}$

Iteration $=40$, Energy $=-74.35807580 \mathrm{Ha}$

Iteration $=60$, Energy $=-74.51446847 \mathrm{Ha}$

Iteration $=80$, Energy $=-74.56593923 \mathrm{Ha}$

Iteration $=100$, Energy $=-74.58052004 \mathrm{Ha}$

Iteration $=120$, Energy $=-74.58713612 \mathrm{Ha}$

Iteration $=140$, Energy $=-74.59031295 \mathrm{Ha}$

Iteration $=160$, Energy $=-74.59175088 \mathrm{Ha}$

Iteration $=180$, Energy $=-74.59237374 \mathrm{Ha}$

Final convergence parameter $=0.00000885 \mathrm{Ha}$

Final value of the ground-state energy $=-74.59262921 \mathrm{Ha}$

Accuracy with respect to the FCI energy: 73.45643975 Ha $(46094.13631472 \mathrm{kcal} / \mathrm{mol})$

Final circuit parameters $=$

[[ 5.54193389e+00 8.41790050e-05 3.08160077e+00]

[ 7.03997361e+00 6.60655067e+00 -3.13026870e+00] 
[ $2.98479079 \mathrm{e}+00-3.12940495 \mathrm{e}+00-3.23993298 \mathrm{e}-01]$

[ $1.28993324 \mathrm{e}+008.01379434 \mathrm{e}-174.56873497 \mathrm{e}+00]$

[ $2.39087053 \mathrm{e}+003.05745058 \mathrm{e}+001.40761930 \mathrm{e}+00]$

[ $1.04826882 \mathrm{e}+003.14163976 \mathrm{e}+00-6.39082649 \mathrm{e}-01]$

[ $9.83531192 \mathrm{e}-01-3.13675958 \mathrm{e}+00-7.99021838 \mathrm{e}+00]$

[ 2.05340338e+00 3.14161455e+00 -2.32943051e+00]]

The ground state energy of oxygen obtained from 100 iterations of optimization is 147.55131990 Ha. and the final circuit parameters and other optimization details are given below.

Iteration $=0$, Energy $=-146.26504092 \mathrm{Ha}$

Iteration $=20$, Energy $=-147.30290612 \mathrm{Ha}$

Iteration $=40$, Energy $=-147.53700197 \mathrm{Ha}$

Iteration $=60$, Energy $=-147.54993831 \mathrm{Ha}$

Iteration $=80$, Energy $=-147.55113346 \mathrm{Ha}$

Iteration $=100$, Energy $=-147.55129411 \mathrm{Ha}$

Final convergence parameter $=0.00000093 \mathrm{Ha}$ 
Final value of the ground-state energy $=-147.55131990 \mathrm{Ha}$

Accuracy with respect to the FCI energy: $146.41513044 \mathrm{Ha}(91875.93359949 \mathrm{kcal} / \mathrm{mol})$

Final circuit parameters $=$

[[ 5.54193389e+00 1.28150550e-03 3.00578101e+00]

[ $7.03997361 \mathrm{e}+006.28265660 \mathrm{e}+00-3.04573222 \mathrm{e}+00]$

[ $2.98479079 \mathrm{e}+001.64453090 \mathrm{e}-14-3.21635666 \mathrm{e}-01]$

[ $1.28993324 \mathrm{e}+00-7.39491055 \mathrm{e}-154.56303441 \mathrm{e}+00]$

[ $2.39087053 \mathrm{e}+001.38077123 \mathrm{e}-07$ 1.39488313e+00]

[ $1.04826882 \mathrm{e}+006.28318512 \mathrm{e}+00-6.93713097 \mathrm{e}-01]$

[ $9.83531192 \mathrm{e}-01-3.13074430 \mathrm{e}+00-8.02415297 \mathrm{e}+00]$

[ $2.05340338 \mathrm{e}+003.12988509 \mathrm{e}+00-2.33843681 \mathrm{e}+00]$

[ 7.13064445e+00 -6.28151756e+00 1.89390706e-01]

[-5.88055408e-01 6.28226080e+00 4.56612645e+00]

[ 4.86781694e-01 3.14159210e+00 -2.83682385e+00]

[-6.22285563e+00 -3.14159216e+00 4.97256496e-01]] 
The ground state energy of hydrogen obtained from 100 iterations of optimization is 1.13615709 Ha. and the final circuit parameters and other optimization details are given below.

Iteration $=0$, Energy $=-0.88179557 \mathrm{Ha}$

Iteration $=20$, Energy $=-1.13380513 \mathrm{Ha}$

Iteration $=40$, Energy $=-1.13558756 \mathrm{Ha}$

Iteration $=60$, Energy $=-1.13585794 \mathrm{Ha}$

Iteration $=80$, Energy $=-1.13600617 \mathrm{Ha}$

Iteration $=100$, Energy $=-1.13608848 \mathrm{Ha}$

Iteration $=120$, Energy $=-1.13613394 \mathrm{Ha}$

Final convergence parameter $=0.00000099 \mathrm{Ha}$

Final value of the ground-state energy $=-1.13615709 \mathrm{Ha}$

Accuracy with respect to the FCI energy: $0.00003237 \mathrm{Ha}(0.02031093 \mathrm{kcal} / \mathrm{mol})$

Final circuit parameters $=$

[[ 5.54193389e+00 1.30219523e-08 3.07479606e+00]

[ 7.03997361e+00 6.28318530e+00 -3.07020901e+00]

[ 2.98479079e+00 -2.09540998e-01 -4.16893297e-02]

[ $1.28993324 \mathrm{e}+001.30907537 \mathrm{e}-124.56873497 \mathrm{e}+00]]$ 
The energy difference between reactants and product for the dissociation of water into hydrogen and oxygen yields the dissociation energy which is comparable to experimental measure and a demonstrated use of Pennylane interface to perform ab initio quantum chemical calculations on near-term quantum devices has been shown.

\section{Conclusion}

Fault-tolerant quantum computers may be far off, however solving real world quantum chemistry problems on near-term quantum devices is possible through the Pennylane which provides interface to use the quantum hardware as provided by quantum hardware providers such as IBM, Google or Microsoft. A demonstrated use of Pennylane to solve real world quantum chemical problems on near-term quantum devices is shown. Ab initio quantum chemical calculations of dissociation energy of water molecule on the near-term quantum hardware using the Pennylane interface yielded a dissociation energy comparable to experiment and therefore it is recommended that the quantum chemistry community use nearterm quantum devices to leverage quantum computing advantage to solve quantum chemical problems.

\section{References}

1. Aleksandrowicz, G., Alexander, T., Barkoutsos, P., Bello, L., Ben-Haim, Y., Bucher, D., Cabrera-Hernández, F.J., Carballo-Franquis, J., Chen, A., Chen, C.F. and Chow, J.M., 2019. Qiskit: An open-source framework for quantum computing. Accessed on: Mar, 16.

2. Cross, A., 2018. The IBM Q experience and QISKit open-source quantum computing software. In APS March Meeting Abstracts (Vol. 2018, pp. L58-003). 
3. MacQuarrie, E.R., Simon, C., Simmons, S. and Maine, E., 2020. The emerging commercial landscape of quantum computing. Nature Reviews Physics, 2(11), pp.596598.

4. Hassija, V., Chamola, V., Saxena, V., Chanana, V., Parashari, P., Mumtaz, S. and Guizani, M., 2020. Present landscape of quantum computing. IET Quantum Communication, 1(2), pp.42-48.

5. Cao, Y., Romero, J., Olson, J.P., Degroote, M., Johnson, P.D., Kieferová, M., Kivlichan, I.D., Menke, T., Peropadre, B., Sawaya, N.P. and Sim, S., 2019. Quantum chemistry in the age of quantum computing. Chemical reviews, 119(19), pp.1085610915.

6. Bergholm, V., Izaac, J., Schuld, M., Gogolin, C., Alam, M.S., Ahmed, S., Arrazola, J.M., Blank, C., Delgado, A., Jahangiri, S. and McKiernan, K., 2018. Pennylane: Automatic differentiation of hybrid quantum-classical computations. arXiv preprint arXiv:1811.04968.

7. Kandala, A., Mezzacapo, A., Temme, K., Takita, M., Brink, M., Chow, J.M. and Gambetta, J.M., 2017. Hardware-efficient variational quantum eigensolver for small molecules and quantum magnets. Nature, 549(7671), pp.242-246.

8. Wang, D., Higgott, O. and Brierley, S., 2019. Accelerated variational quantum eigensolver. Physical review letters, 122(14), p.140504.

9. Wei, S., Li, H. and Long, G., 2020. A full quantum eigensolver for quantum chemistry simulations. Research, 2020.

10. Sun, Q., Berkelbach, T.C., Blunt, N.S., Booth, G.H., Guo, S., Li, Z., Liu, J., McClain, J.D., Sayfutyarova, E.R., Sharma, S. and Wouters, S., 2018. PySCF: the Python-based simulations of chemistry framework. Wiley Interdisciplinary Reviews: Computational Molecular Science, 8(1), p.e1340. 